\title{
First principles calculation of electronic properties and effective mass of zinc-blende GaN
}

\author{
Guangqi Xie, and Huanyou Wang* \\ Academy of Electronic Information and Electrical Engineering, Xiangnan University, Chenzhou \\ 423000, China
}

\begin{abstract}
Based on the first principle pseudopotential plane wave method, the electronic structure of zinc-blende semiconductor $\mathrm{GaN}$ is calculated. Using the relativistic treatment of valence states, the spin orbit splitting energy of valence band top near the center of Brillouin region is calculated. Based on the effective mass approximation theory, the effective mass of electrons near the bottom of the conduction band and the effective mass of light and heavy holes near the $\Gamma$ point along the directions of [100], [110] and [111] are calculated. These parameters are valuable and important parameters of optoelectronic materials.
\end{abstract}

Keywords: Zinc-blende GaN, Electronic structure, Effective-mass, First principle.

\section{Introduction}

Zinc-blende structure semiconductor materials are not only widely used in light-emitting diodes, laser diodes, photoelectric modulators and photodetectors, but also the basic materials for making heterostructures, superlattices and quantum wells. The study of the energy band and effective mass of the crystal can provide theoretical basis and experimental guidance for the development of new semiconductor materials, such as quantitative explanation of the photoelectric conversion efficiency, carrier mobility, acceptor impurities and excitation energy levels related to the degenerate state at the top of the valence band. In recent years, Ramos L E [1] group and Suzuki m [2] et al. using the full potential linear augmented plane wave method (FLAPW), Fan W J [3] using the empirical pseudopotential method, and Ahn D [4] using the k.p method calculated the basic energy gap and effective mass of zinc-blende GaN. In terms of experiments, Perlin [5] team measured the effective mass of electrons with reflection spectrum and Hall effect, and Gass M H [6], Shokhovets S [7] measured the effective mass of electrons with different experimental methods. However, Perlin's indirect experimental results are questioned, because the best method to measure the effective mass of electrons is cyclotron resonance technology, and the Hall effect can not obtain the required high mobility and high magnetic

*Corresponding author: wanghy@xnu.edu.cn 
field; In addition, the defect and impurity of the sample itself will highly affect the band edge reflectivity and absorptivity. Based on the first principle, the energy band structures near the valence band top (VBM) and conduction band bottom (CBM) of zinc-blende GaN are calculated by using the pseudopotential plane wave method (PP-PAW). Combined with the effective mass approximation theory, the effective masses of electrons and holes near the center of Brillouin zone are obtained.

\section{Theoretical method}

Based on the density functional theory and the first principle pseudopotential plane wave method, this paper constructs the exchange correlation term in the single electron potential by using the local density approximation (LDA) in reference [8], generates the mode conservation pseudopotential by using the method in reference [9], and self consistently solves the Kohn sham equation to obtain the energy band structure of zinc-blende GaN. Because Ga-3 $\mathrm{d}^{10}$ has high electron energy, relatively local characteristics, and has strong hybridization with $\mathrm{N}-2 \mathrm{~s}^{2}$ electrons, which plays an important role in the description of energy band, Ga-3d $d^{10}$ is treated as valence state like Ga- $4 s^{2} 4 p^{3}$ and $N-2 s^{2} 2 p^{1}$. The charge density is calculated by tetrahedral integration, and the corresponding integration region is reduced Brillouin zone, which is calculated as $8 \times 8 \times 8$ spatial grid. At the same time, considering the large mass of $\mathrm{Ga}$ atom, the valence state is treated by relativistic effect in the calculation. By calculating the dispersion spectra in several high symmetry directions in the Brillouin region, the calculation of electron band structure is connected with the effective mass, which is valence band fitting. Because in $\Gamma$ point CBM belongs to the non degenerate zone, the parabolic approximation method is used to obtain the electron effective mass. The convergence criterion of this calculation takes the square remainder of wave function $1 \times 10^{-12}$.

\section{Results and analysis}

\subsection{Structure and electronic properties}

The space group of zinc-blende GaN is F-43M (216), which is composed of cations and anions in face centered cubic structure along the space diagonal. In this paper, the optimized theoretical lattice constant is adopted. Since there is only one lattice constant, the convergence test of monkhorst pack lattice and truncation energy is only required by using the principle of minimum energy. The calculated theoretical lattice constant is $4.552 \AA$. Compared with the experimental values of 4.50 [10] and 4.531 [11], the error is only $1 \%$.

Based on the calculated theoretical lattice parameters, the dispersion curves of several main symmetry directions in the first Brillouin region are calculated, and the spin orbit interaction is considered at the same time. The calculated fundamental band gap energies $\mathrm{Eg}$ and valence bandwidth $\Delta \mathrm{Ev}$ are $1.83 \mathrm{eV}$ and $17.07 \mathrm{eV}$ respectively. In general, our calculated values are in good agreement with calculated values in references [1] and [2], which adopted FLAPW method, but there is a large error compared with the experimental fundamental band gap energies $3.21 \mathrm{eV}$ [12] and $3.3 \mathrm{eV}$ [3]. This is due to the shortcomings of LDA method in solving the excited state energy and ignoring the nonlocal characteristics of exchange correlation potential. However, the properties of the local structure of the system concerned in this paper will not be affected by this error. 

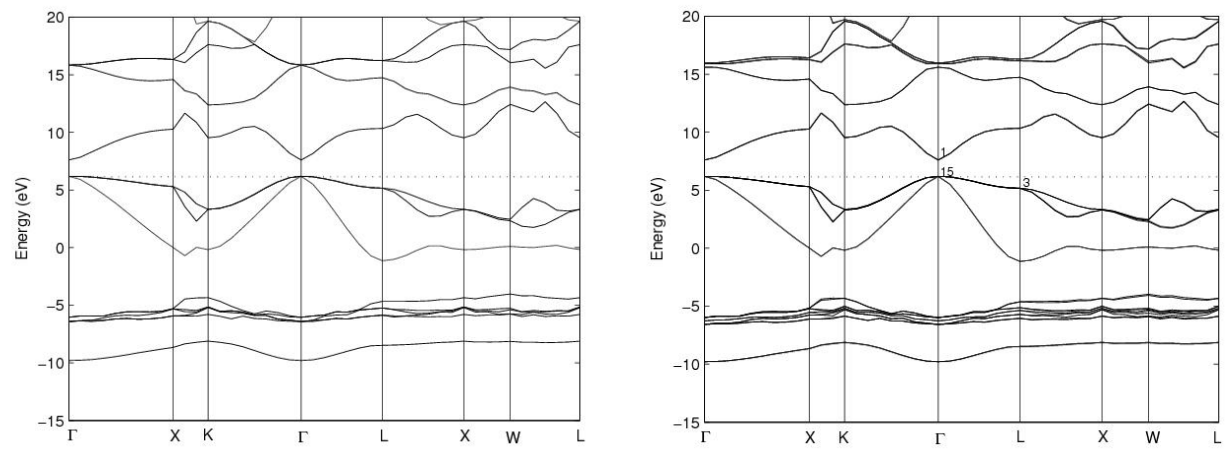

Fig. 1. Energy band structure of zinc-blende semiconductor material GaN. Relativistic effect is not considered in the left figure and relativistic effect is considered in the right figure.

Due to the spin-orbital interaction, the top $\Gamma_{15}$ of the valence band in Brillouin region splits into $\Gamma_{8}$ degenerate states and $\Gamma_{7}$ degenerate states, and their splitting energy $\Delta_{s o}$ is $16 \mathrm{meV}$. In order to further understand the electron combination in the energy interval, the total density of states and the fractional density of states distributions are calculated considering the spin-orbit interaction. As can be seen from figure 2, the band structure of zinc-blende structure is mainly composed of four parts, namely, three electron filled bands (valence band) and one non-electron filled band (conduction band) with the highest energy. The filling band with the lowest energy corresponds to $-9.88 \mathrm{eV}$ to $-8.14 \mathrm{eV}$ is mainly composed of the $2 \mathrm{~s}$ electron states of $\mathrm{N}$ and the $4 \mathrm{~s}$ states of $\mathrm{Ga}$. The electron filling band in the energy center is located between $-6.45 \mathrm{eV}$ and $-4.10 \mathrm{eV}$. This band region clearly shows the local characteristics of $3 \mathrm{~d}$ electron state for $\mathrm{Ga}$, indicating that $\mathrm{Ga}$ atom becomes the negative center. The highest energy electron filled band is between $-1.08 \mathrm{eV}$ and $6.19 \mathrm{eV}$. The low energy part of the band is mainly composed of Ga 4 s state and N $2 p$ state, and the high energy part of the band is composed of Ga $4 p$ state and $N 2 p$ state. The band with the highest energy is called the conduction band, and CBM is located at the point with a value of $8.03 \mathrm{eV}$. On the low energy side of the conduction band, except for $\mathrm{Ga} 3 \mathrm{~d}$, the contribution of the other four fractions is not much different.

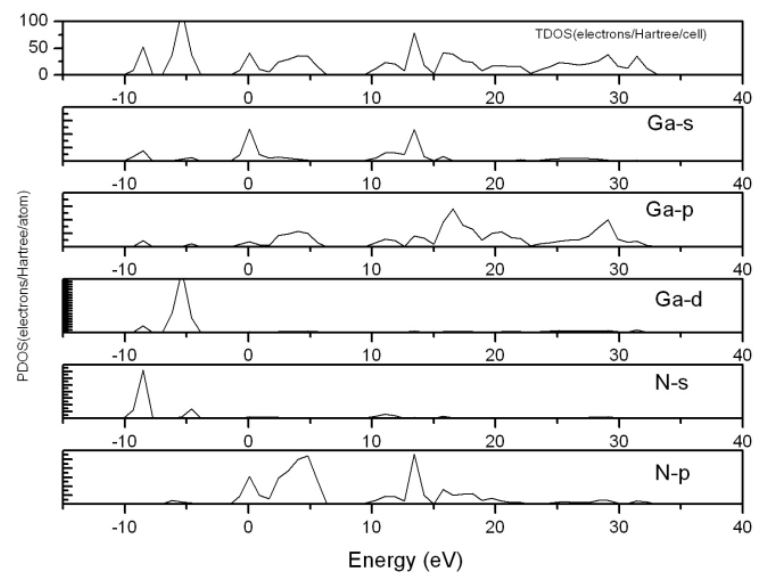

Fig. 2. Total and partial density of state of zinc-blende GaN. 


\subsection{Effective mass}

The effective mass of electrons and holes is different from the real mass of electrons and holes, and their size depends on the electronic structure of the very small region of $\mathrm{K}$ space, or more specifically, on the region near Brillouin zone. The effective mass determines their inertia and mobility and has a direct effect on the optical phenomena and electron transport of semiconductor materials. Firstly, the relationship between effective mass and energy band is introduced:

Substitute the Bloch wave function $\Psi_{k}(\vec{r})=\exp (i \vec{k} \cdot \vec{r}) u_{k}(\vec{r})$ into the energy eigenequation,

$$
\left(\frac{p^{2}}{2 m}+\frac{\hbar \vec{k} \cdot \vec{p}}{m}+\frac{\hbar^{2} k^{2}}{2 m}+V\right) u_{n k}=E_{n k} u_{n k}
$$

For nondegenerate band, the wave function and energy eigenvalue of the wavelet vector $\mathrm{k}$ point can be obtained by solving the equation (1),

$$
E_{n k}=E_{n, k_{0}}+\frac{\hbar^{2} k^{2}}{2 m}+\frac{\hbar^{2}}{m^{2}} \sum_{n^{\prime} \neq n} \frac{\left|\left\langle u_{n 0}|k \cdot p| u_{n^{\prime} 0}\right\rangle\right|^{2}}{E_{n 0}-E_{n^{\prime} 0}}
$$

Here, $m^{*}$ satisfies the following equation,

$$
\frac{1}{m^{*}}=\frac{1}{m}+\frac{2}{m^{2} k^{2}} \sum_{n^{\prime} \neq n} \frac{\left|\left\langle u_{n 0}|k \cdot p| u_{n^{\prime} 0}\right\rangle\right|^{2}}{E_{n 0}-E_{n^{\prime} 0}}
$$

For degenerate bands, the coupling between degenerate bands needs to be treated by perturbation method. The energy variation between arbitrary wave vectors is obtained by diagonalizing the matrix using k.p [16] method,

$$
H_{i j}^{\prime}=\left\langle\psi_{i}\left|\frac{\hbar^{2} k^{2}}{2 m}+\frac{\hbar k \cdot p}{m}\right| \psi_{j}\right\rangle+\sum_{j} \mid\left\langle\psi_{i}\left|\frac{\hbar^{2} k^{2}}{2 m}+\frac{\hbar k \cdot p}{m}\right| \psi_{j}\right\rangle^{2} \frac{1}{E_{i}-E_{j}}
$$

In general, the effective mass is a tensor related to band structure,

$$
\frac{1}{m_{i j}^{*}}=\frac{1}{\hbar^{2}} \frac{\partial^{2} E_{k=0}}{\partial k_{i} \partial k_{j}}
$$

According to the effective mass approximation theory, the energy band of the semiconductor material with the symmetrical structure of zinc-blende near CBM is a parabola, a non-degenerate band, with s-like characteristics and slight anisotropy. Therefore, the average value of the effective mass of the electron in different $k$ directions can be taken as the effective mass of the electron near CBM. VBM splits from the original six-fold degenerate state into four-fold degenerate state and double degenerate state after taking into account spin-orbital coupling, and the total Hamiltonian is decomposed into k.p part of Hk.p and the spin-orbital interaction Hso. If the light and heavy hole valence band states are respectively represented by $\left|\begin{array}{ll}j & m_{j}\end{array}\right\rangle$, by calculation, for the quadruple degenerate states $\Gamma_{8 v}$ has $\left|\frac{3}{2}, m_{3 / 2}\right\rangle$, here $m_{3 / 2}= \pm \frac{3}{2}, \pm \frac{1}{2}$. For doubly degenerate states $\Gamma_{7 v}$ has $\left|\frac{1}{2}, m_{1 / 2}\right\rangle$, here $m_{1 / 2}= \pm \frac{1}{2}$. With the above states, the total $6 \times 6 \mathrm{LK}$ Hamiltonian matrix can be expressed as 


$$
\left(\begin{array}{cccccc}
Q & S & R & 0 & \frac{i}{\sqrt{2}} S & -i \sqrt{2} R \\
S^{*} & T & 0 & R & -\frac{i}{\sqrt{2}}(Q-T) & i \sqrt{\frac{3}{2}} S \\
R^{*} & 0 & T & -S & -i \sqrt{\frac{3}{2}} S^{*} & -\frac{i}{\sqrt{2}}(Q-T) \\
0 & R^{*} & -S^{*} & Q & -i \sqrt{2} R^{*} & -\frac{i}{\sqrt{2}} S^{*} \\
-\frac{i}{\sqrt{2}} S^{*} & \frac{i}{\sqrt{2}}(Q-T) & i \sqrt{\frac{3}{2}} S & i \sqrt{2} R & \frac{1}{2}(Q+T)-\Delta_{S O} & 0 \\
i \sqrt{2} R * & -i \sqrt{\frac{3}{2}} S * & \frac{i}{\sqrt{2}}(Q-T) & \frac{i}{\sqrt{2}} S & 0 & \frac{1}{2}(Q+T)-\Delta_{S O}
\end{array}\right)
$$

Because the zinc-blende structure is a direct band gap semiconductor, we are only interested in the effective mass of electrons and holes near the center of the Brillouin region. By using parabolic approximation method, the effective mass of the electron in the range of $5 \%$ along the high symmetry direction is calculated respectively. Take 40 points in each direction, and by fitting, get the effective mass of the electron near $\Gamma$ point along the $\Delta$ axis, $\Sigma$ axis and $\Lambda$ axis, and the average value is taken as the effective mass of electrons near CBM. In order to calculate the effective mass of holes, six eigenvalues are diagonalized for formula (6) firstly, then the least square method is used to fit the dispersion relation near VBM, and the parameter expression of Luttinger is used to obtain the effective mass of light and heavy holes along the directions of [100], [110] and [111]. The calculated results and the theoretical and experimental values of other literatures are listed in table 1 . The calculated electron effective mass is in good agreement with the experimental values of literature [13].

Table 1. Effective masses of electrons and holes in (100), (110) and (111) for zinc-blende GaN.

\begin{tabular}{|c|c|c|c|c|c|c|c|c|}
\hline & $m_{e}$ & $m_{h h}^{(100)}$ & $m_{l h}^{(100)}$ & & $m_{h h}^{(111)}$ & $m_{l h}^{(111)}$ & $m_{h h}^{(110)}$ & $m_{l h}^{(110)}$ \\
\hline & 0.17 & 0.90 & 0.23 & 2.22 & 0.18 & 1.60 & 0.22 & \\
\hline$[14]^{\mathrm{a}}$ & 0.17 & 0.85 & 0.24 & & & & & \\
\hline$[1]^{\mathrm{b}}$ & 0.14 & 0.86 & 0.21 & 2.09 & 0.19 & 1.65 & 0.19 & \\
\hline$[6]^{\mathrm{c}}$ & 0.20 & & & & & & & \\
\hline$[7]^{\mathrm{d}}$ & 0.18 & & & & & & & \\
\hline$[13]^{\mathrm{e}}$ & 0.15 & & & & & & & \\
\hline
\end{tabular}

As can be seen from table 1, it's going to be $m_{h h}>m_{l h}>m_{e}^{*}$ in either direction. This is because the effective mass of the carrier depends solely on the energy spectrum. It can be seen from the band diagram that the curvature radius of the heavy cavity zone near VBM is obviously larger than that of the light cavity zone, making the effective mass of the heavy cavity several times or even ten times that of the light cavity. Because the radius of curvature of CBM is different along the directions of [100], [110] and [111], the effective mass of the hole is anisotropic, especially the effective mass of the heavy hole has a large difference, while the effective mass of the light hole has a small difference.

\section{Conclusion}

In this paper, the structure and electronic properties of the semiconductor material $\mathrm{GaN}$ with zinc-blende structure are calculated by using the first principle PP-PAW in LDA. The calculated lattice constants, band gaps and spin-orbit splitting energies are in good 
agreement with those obtained by other theoretical and experimental methods. Based on the effective mass approximation theory, the dispersion relation between CBM and VBM near the center point of Brillouin region is fitted, and some important parameters which are valuable for photoelectronic materials are obtained. For example, the effective mass $m_{e}^{*}$ of the electron near CBM, and the effective mass $m_{l h}$ and $m_{h h}$ of the light and heavy holes near point $\Gamma$ along the directions of [100], [110] and [111]. These effective masses and orbital splitting energy can provide important theoretical help for other systems such as quantum wells and superlattices.

This work was supported by Science Foundation of Education Department of Hunan Provincial (18C1013)

\section{Reference}

1. Ramos L E, Teles L K, Scolfaro L M R, et al. 2001 Phys. Rev. B 63165210

2. Suzuki M and Uenoyama T 1996 Appl. Phys. Lett. 693378

3. Fan W J, Li M F, and Chong T C 1996 J. Appl. Phys. 79188

4. Doyeol Ahn 1996 J. Appl. Phys. 797731

5. Perlin P and Litwin-Staszewska E 1996 Appl. Phys. Lett. 661114

6. Gass M H, Papworth A J, and Beanland R, et al. 2006 Phys. Rev. B 73035312

7. Shokhovets S, Ambacher O and Gobsch G 2007 Phys. Rev. B 76125203

8. Perdew J P and Zunger A 1991 Phys. Rev. B 235048

9. Hartwigsen C, Goedecker S, and Hutter J 1998 Phys. Rev. B 583641

10. Lei T and Moustakas T D 1992 J. Appl. Phys. 714933

11. Kim K, Lambrecht W R, and Segall 1996 Phys. Rev. B 5316310

12. Powell R C, Lee N-E, and Greene 1993 J. Appl. Phys. 73189

13. Fanciulli T, Lei T, and Moustakas T D 1993 Phys. Rev. B 4815144

14. Gass M H, Papworth A J, and Joyce T B, et al. 2004 Appl. Phys. Lett. 841453 